\title{
USO DE BIOCARBÓN DE CÁSCARAS DE SEMILLA DE PALMA ACEITERA ENRIQUECIDO EN BIOL DE ESTIÉRCOL DE VACA PARA MEJORAR SUELOS SOBREPASTOREADOS
}

\section{Use of biochar from oil palm kernel shells enriched in cow manure biol to improve overgrazed soils}

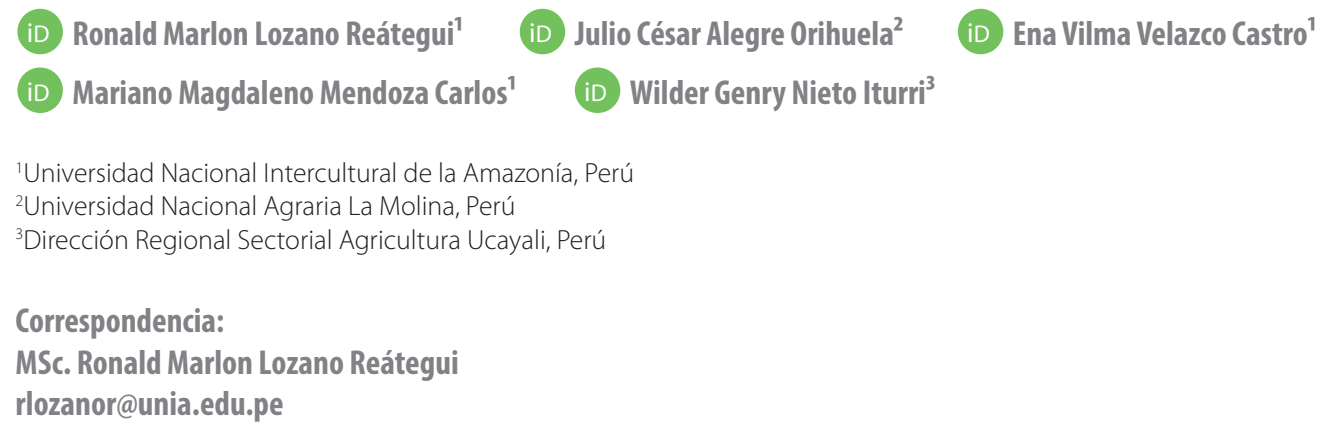

\section{RESUMEN}

Las agroindustrias de aceite de palma (Elaeis guineensis) en Pucallpa, producen como residuos, cáscaras de semillas (cuescos) contaminando el suelo; la crianza de ganado vacuno, origina estiércol (vacaza), que produce gases contaminantes; $y$, el deterioro de suelos por sobrepastoreo, son problemas ambientales que necesitan soluciones viables. La presente investigación evaluó el mejoramiento las propiedades fisicoquímicas de los suelos degradados por sobrepastoreo, aplicando biocarbón fabricado de cuescos por pirólisis y enriquecido en biol de vacaza elaborado por fermentación anaeróbica. La metodología comprendió un Diseño en Bloques Completamente al Azar (DBCA) con un control (T0) y tres tratamientos (T1, T2, T3), con 5, 10 y $15 \mathrm{t} \mathrm{h}^{-1}$ de biocarbón enriquecido respectivamente, asignados aleatoriamente por triplicado a 16 unidades

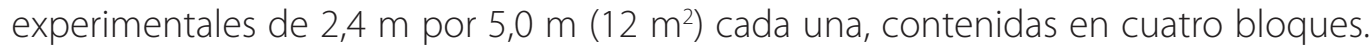
El análisis estadístico comprendió la prueba Shapiro-Wilks y Levenne, también Tukey y Friedman. Los resultados para pH, materia orgánica, densidad aparente, capacidad de intercambio catiónico y nitrógeno no resultaron significativos ( $p>0,05)$, en cambio el fósforo con T3 y Potasio con T2, si mostraron diferencia significativa $(p<0,05)$, para mejorar las características fisicoquímicas de los suelos sobrepastoreados por aplicación de biocarbón enriquecido con biol de vacaza.

Palabras clave: Biocarbón, biol, pirólisis, fermentación anaeróbica.

\section{ABSTRACT}

The palm oil (Elaeis guineensis) agroindustries in Pucallpa produce as residues, seed shells (cuescos) contaminating the soil; the raising of cattle, originates manure (vacaza), that produces polluting gases; and, the deterioration of soils by overgrazing, are environmental problems that need viable solutions. The present investigation evaluated the improvement of the physicochemical properties of degraded soils 
by overgrazing, applying biochar made from pyrolysis pits and enriched in cow biol produced by anaerobic fermentation. The methodology comprised a Completely Random Block Design (DBCA) design with a control (T0) and 3 treatments (T1, T2, T3), with 5,10 and $15 \mathrm{th}^{-1}$ of enriched biochar respectively, randomly assigned in triplicate to 16 experimental units of $2,4 \mathrm{~m}$ by $5,0 \mathrm{~m}\left(12 \mathrm{~m}^{2}\right)$ each, contained in four blocks. Statistical analysis included the Shapiro-Wilks and Levenne test, also Tukey and Friedman. The results for $\mathrm{pH}$, organic matter, apparent density, cation exchange capacity and nitrogen were not significant ( $p>0.05$ ), whereas phosphorus with T3 and potassium with T2 did show a significant difference $(p<0.05)$ to improve the physicochemical characteristics of overgrazed soils by applying biochar enriched in cow biol.

Key words: Biochar, biol, pyrolysis, anaerobic fermentation.

\section{INTRODUCCIÓN}

La crisis ambiental, se origina en la mayoría de los casos por la mala o ineficiente gestión que se hace de los desechos contaminantes. El desarrollo de la región Ucayali, se está logrando por el impulso de empresas agroindustriales, que otorgan valor agregado a las materias primas de origen agrícola, cuyo proceso de transformación, como es el caso de la palma aceitera (Elaeis guineensis), produce residuos sólidos (rastrojos y cuescos), líquidos (efluentes con aceite residual) y gaseosos (humos y vapores), que, al no ser tratados, cada día van acumulándose de modo preocupante en el ambiente.

La crianza de ganado vacuno, presenta dos problemáticas bien marcadas: producción de estiércol y suelos sobrepastoreados, así, el estiércol, se descompone en gas metano $\left(\mathrm{CH}_{4}\right)$, originado a nivel global entre el 5-20\% por la ganadería bovina (Huaraya y Sancho, 2020), posee 25-28 veces el potencial de calentamiento global con respecto al $\mathrm{CO}_{2}$ (Hernández-Medrano y Medrano, 2018); y, el sobrepastoreo, que por efecto de los cascos del ganado, producen la pérdida de la vegetación, exponiendo al suelo a la acción erosiva de la lluvia, el sol y el viento, perdiendo así su capa fértil y capacidad agrícola. El concepto de Economía Circular, enfocado en producirsin contaminación (Sandoval etal., 2017), está impulsando a aprovechar los residuos, para convertirlos en productos útiles, así, los purines y estiércoles de ganado vacuno con ciertos insumos, son transformados en fertilizantes líquidos (bioles), mediante fermentación anaeróbica (Crespo, 2019) desarrollada por microorganismos que descomponen los elementos nutritivos de los residuos, produciendo fertilizantes, que agregados al suelo y las plantas, mejoran su capacidad agrícola e incrementan la productividad vegetal.

Los residuos lignocelulósicos como la cascarilla de arroz, son transformados en biocarbón por pirólisis (Klug, 2012), en Tailandia, mediante el Carbonizador Estilo Abierto "PhilRice" (Hugill, 2013), extendiéndose a otros tipos de forrajes como: mazorcas y hojas de maíz, cáscara de coco, residuos de la industria de la palma aceitera, bagazo de caña de azúcar, etc., tecnología apropiada para la transformación de los cuescos en biocarbón.

La recuperación de la capacidad agrícola de suelos degradados, utilizando biocarbón ya ha sido investigado por Arévalo (2020), quien experimentó en un suelo degradado por sobrepastoreo, cuatro tratamientos: Biocarbón (T1), elaborado de acícula de pino (Pinus patula), gallinaza (T2) y eco abonaza (T3), y un testigo sin abono (T4); también Obregón (2019), experimentó biocarbón de excretas de pollo y maleza de higuera, usando dosis de $5 \%$ (T1), 10 $\%$ (T2), $15 \%$ (T3) y 0 \% (T control), para disminuir la salinidad de un suelo degradado.

El sobrepastoreo, disminuye la calidad del suelo, conllevando a generar limitaciones significativas e importantes para su uso y productividad, elementos claves, para la sostenibilidad de la agricultura en los trópicos, haciendo necesario 
considerar iniciativas que busquen solucionar este problema, por lo cual, se planteó como objetivo general, evaluar la aplicación de biocarbón de cáscaras de semilla de palma aceitera enriquecido en biol de estiércol de vaca, para mejorar suelos sobrepastoreados en Ucayali.

\section{MATERIALES Y MÉTODOS Lugar de ejecución}

El estudio se desarrolló en las instalaciones del fundo "El Cedro"-Patoyacu, ubicado en el Asentamiento Humano "Acho Mego", del distrito de Yarinacocha, provincia de Coronel Portillo, región Ucayali, mostrando como coordenadas: 18L 454829 y UTM 9077178.

\section{Descripción y características edafoclimáticas del área de estudio}

El área de estudio, presentó un suelo con características de compactación por efecto del sobrepastoreo. La clasificación de la región corresponde al ecosistema de bosque tropical semi siempre verde estacional (Cochrane, 1982), y según la estación meteorológica de la Universidad Nacional de Ucayali, las condiciones climáticas promedio anuales en la ciudad de Pucallpa son: temperatura media anual de $26,9^{\circ} \mathrm{C}$, con una máxima de $36,5^{\circ} \mathrm{C}$ y una mínima de 17,4 ${ }^{\circ} \mathrm{C}$; precipitación promedio anual de 1773 mm; brillo solar de 159,1 h y H.Ro 83,8\% (Flores et al., 2020).

\section{Diseño de la investigación}

Se trató de una investigación de Diseño en Bloques Completamente al Azar (DBCA) con cuatro repeticiones, tres tratamientos y un control, por lo cual el estudio presentó cuatro bloques compuestos cada uno por cuatro parcelas como unidades experimentales.

\section{Elaboración del biol y análisis de nitrógeno}

El estiércol de ganado vacuno fue recolectado de un establo lechero y transportado hacia el lugar de elaboración. El estiércol en una cantidad de $12,7 \mathrm{~kg}$ se depositó en el interior de una galonera de $50 \mathrm{~L}$, agregando a continuación, 10 $\mathrm{g}$ de levadura para pan, $2 \mathrm{~L}$ de suero de queso, medio litro (0,5 L) de jugo de caña de azúcar, 400 g de ceniza y 24,5 L de agua. La mezcla se dejó fermentar por 84 días, tiempo en que se logró transformar los residuos en biol, cuyo punto final, se probó con un poco de alcohol de $96^{\circ}$, que se agregó a una muestra del biol, formándose una capa blanca en su superficie, indicio de la formación de nutrientes y componentes fitohormonales. Posteriormente, el biol fue analizado en su contenido de nitrógeno en el Laboratorio de Análisis de Aguas y Alimentos de la Empresa Natura Analítica S.A.C. de Pucallpa.

\section{Fabricación del biocarbón, enriquecimiento y análisis}

Los cuescos de palma aceitera fueron transportados de la planta hasta el terreno experimental, donde fueron secados al sol por tres días, luego sometidos a pirólisis en carbonizadores tipo PhilRice estilo abierto. Después de ocho horas de carbonización en rangos de temperatura entre 353,0 y $576,6^{\circ} \mathrm{C}$, fue enfriado, envasado, molido y tamizado a tamaños de $\leq 1,0 ; 1,4$ y $2,0 \mathrm{~mm}$, y, en función de la cantidad obtenida de cada tamaño, se formuló la mezcla: 2,5 kg de $\leq 1,0$ (42\%), 1,4 $\mathrm{kg}$ de $1,4 \mathrm{~mm}(23 \%)$ y $2,1 \mathrm{~kg}$ de $2,0 \mathrm{~mm}$ (35\%), haciendo un total de $6,0 \mathrm{~kg}$ de biocarbón, que fue mezclado con biol diluido de vacaza (1 parte de biol: 9 partes de agua) y dejado en reposo ocho días para su activación, y luego fue usado como enmienda. Posteriormente, se caracterizó fisicoquímicamente al biocarbón y biocarbón enriquecido en el Laboratorio de Análisis de Suelos, Plantas, Aguas y Fertilizantes-LASPAF de la Universidad Nacional Agraria La Molina-UNALM, en los cuales se determinó: humedad $(H), p H$, conductividad eléctrica (CE), materia orgánica $(\mathrm{MO})$, carbono $(\mathrm{C})$, nitrógeno $(\mathrm{N})$, fósforo $(\mathrm{P})$, potasio $(K)$ cuyos valores obtenidos se muestran en la Tabla 1.

\section{Establecimiento de la parcela experimental y análisis del suelo}

Luego de delimitar el terreno experimental, se definió los cuatro bloques en función de la pendiente que mostró el terreno, fueron establecidos las cuatro parcelas de $2,4 \mathrm{~m}$ de ancho y $5 \mathrm{~m}$ de largo $\left(12 \mathrm{~m}^{2}\right)$ dentro de cada bloque, se asignaron aleatoriamente los tratamientos a cada 
parcela, se procedió a obtener las muestras de los suelos, los cuales fueron remitidos al LASPAF de la UNALM, para analizar pH, materia orgánica (MO), densidad aparente (DA), capacidad de intercambio catiónico $(\mathrm{CIC})$, nitrógeno $(\mathrm{N})$, fósforo $(P)$, potasio $(K)$ y clase textural.

\section{Aplicación de la enmienda y análisis del suelo}

Tras ocho días de activación del biocarbón, se agregó al suelo de las parcelas, de acuerdo a la asignación aleatoria de los tratamientos, 6 kg de biocarbón remojado en 15 litros de biol diluido como T1 $\left(5 \mathrm{t} \mathrm{h}^{-1}\right), 12 \mathrm{~kg}$ de biocarbón en 30 litros de biol diluido como T2 $\left(10 \mathrm{t} \mathrm{h}^{-1}\right), 18 \mathrm{~kg}$ de biocarbón en 45 litros de biol diluido como T3 $\left(15 \mathrm{t} \mathrm{h}^{-1}\right)$, y un tratamiento control T0 $\left(0 \mathrm{t} \mathrm{h}^{-1}\right)$; se esperó dos días para la adaptación del consorcio microbiano a las condiciones del suelo, antes de las siembras. Transcurridos 80 días, se tomó muestras de suelo de cada parcela y se envió al LASPAF de la UNALM, para su respectivo análisis.

\section{Análisis estadístico del efecto de la enmienda en las características del suelo}

Se determinó la normalidad de los datos con la prueba de Shapiro-Wilks y la homogeneidad de varianzas con la prueba de Levenne y en los que cumplieron los supuestos, se realizó el análisis de varianza con la prueba Tukey $(p<0,05)$ y en los que no cumplieron, se realizó la prueba de Friedman. Los análisis estadísticos se realizaron con el software InfoStat, versión 2020e.

\section{RESULTADOS Y DISCUSIÓN Elaboración del biol y análisis de nitrógeno}

El biol formulado con estiércol de ganado vacuno, levadura para pan, suero de queso, jugo de caña de azúcar, ceniza y agua, se obtuvo luego de 84 días de fermentación anaeróbica, presentó un contenido de nitrógeno superior al 0,2 \% reportado por Langone et al. (2017) para estiércol fresco de vacuno, debido a que el nitrógeno total del biol se incrementó por el nitrógeno generado en la descomposición microbiana de las proteínas del suero de queso, usado como insumo. La influencia directa del contenido de nitrógeno de la materia prima en el nitrógeno del biol, fue demostrado por Suquilanda (1996), quien determinó 1,6 \% de nitrógeno en biol de estiércol de vacuno alimentado con $60 \%$ de alfalfa, $30 \%$ de maíz ensilado y $10 \%$ de alimento concentrado, y, cuando preparó biol con el estiércol obtenido con la misma alimentación pero agregando alfalfa picada, obtuvo 2,7 \% de nitrógeno, valores que están por encima del determinado en la presente investigación, atribuido al contenido de proteína de la alfalfa fresca (20,14 \%; Marín, 2019), que al descomponerse microbiológicamente, contribuyó a incrementar el nitrógeno del biol. Del mismo modo, Pérez (2009), preparó biol a partir de vacaza al que agregó leche fresca, jugo de caña, roca fosfórica, sulfato de cobre, kudzu fresco, dolomita y agua, obteniendo un biol con 0,35 \% de nitrógeno, valor por debajo del obtenido en este estudio, aun cuando en esta formulación los insumos muestren altos contenidos de proteínas; sin embargo, el porcentaje de nitrógeno en el fertilizante es menor, lo cual se puede explicar debido a que hay una influencia mayor, cuando el nitrógeno y los aminoácidos que ayudarán a aportar nitrógeno provienen del mismo estiércol, en el cual, al parecer ya están más disponibles para la biosíntesis microbiana del nitrógeno del biol. Con respecto al tiempo de fermentación, Rojas-Pérez et al. (2020), elaboró biol de estiércol seco de bovino al cual agregó melaza, levadura, ceniza, hojas de fabáceas, cal y agua, manteniendo por 60 días la fermentación, con lo cual obtuvo un biol con 1,02 \% de nitrógeno, valor que supera al obtenido en esta investigación, mostrando la dificultad de la estandarización del contenido de nitrógeno de los bioles, pues las materias primas tienen diferente procedencia, composición nutricional y tiempo de descomposición o digestión.

\section{Fabricación del biocarbón, enriquecimiento y análisis}

Los cuescos de palma en su composición química según García, et al. (2008), poseen celulosa (30,28 $\%)$, hemicelulosa $(12,72 \%)$ y lignina $(49,58 \%)$, los cuales se descomponen en el proceso de pirólisis, iniciando con la hemicelulosa (180$\left.285^{\circ} \mathrm{C}\right)$, luego la celulosa $\left(275-365^{\circ} \mathrm{C}\right)$, y al final la lignina $\left(365-500^{\circ} \mathrm{C}\right)$ (Tzvetkov et al., 2016), que tienen una gran influencia directa en la 
capacidad adsorbente del biocarbón (Kim et al., 2013), es decir retener nutrientes por adsorción electrostática (Ippolito et al., 2015) en su área superficial, pues juntamente con la temperatura (>500 $\mathrm{C}$ ) y método de pirólisis (Kim et al., 2013), influyen en el tipo de estructura porosa formada en su interior, así según Uribe (2020): macroporos (tamaño > 50 $\mu \mathrm{m}$ ), mesoporos (tamaño entre 2 y $50 \mu \mathrm{m}$ ) y microporos (tamaño $<2 \mu \mathrm{m}$ ), que tienen gran influencia para retener los nutrientes del biol y el consorcio microbiano, pues dichas estructuras serán las que servirán de hospederos de la microbiota, responsable de realizar el trabajo simbiótico juntamente con las raíces de las plantas para el intercambio de nutrientes, pues Plant Health Cure BV (2017), manifiesta que las bacterias (Rizobacterias) y hongos (Micorrizas) entregan fosfatos $\left(\mathrm{PO}_{4}{ }^{3}\right)$, nitratos $\left(\mathrm{NO}_{3}{ }^{-}\right)$, minerales $\left(\mathrm{K}^{+}\right)$y las raíces devuelven azúcares (glucosa- $\mathrm{C}_{6} \mathrm{H}_{12} \mathrm{O}_{6}$ ), promoviendo así el desarrollo fisiológico de las plantas en el suelo intervenido con la enmienda conteniendo el biocarbón que retiene los nutrientes del biol en su estructura porosa exterior e interior.

El enriquecimiento del biocarbón, buscó mejorar sus características fisicoquímicas y nutricionales,
(Tabla 1), aprovechando los nutrientes del biol de vacaza, mediante su inmersión en biol diluido, práctica que algunos autores denominan "maduración" (Hugill, 2013) y "compostaje" (Prost et al., 2013). La "maduración", según Hugill (2013), evita el "shock de nitrógeno" o la adsorción rápida del nitrógeno disponible del suelo por el biocarbón "fresco", limitando su disponibilidad para las plantas, haciendo necesario tratar el biocarbón primero con orina o ser mezclado con compost y/o mojado en té de compost y mezclas de microorganismos indígenas (MOI), para luego ser combinado con el suelo del sitio donde va a ser colocado y dejado en reposo; este descanso permite que el biocarbón adsorba (en su superficie) y absorba (en sus poros internos) todo el nitrógeno disponible hasta el punto de saturación, que dará comienzo el proceso de colonización por los microorganismos, obteniendo como resultado de esta práctica, que el biocarbón maduro, en el suelo, empieza a funcionar casi inmediatamente. El "compostaje", considerado por Prostet al. (2013), modifica la superficie del biocarbón por efecto de la oxidación biótica (alta actividad microbiológica) y abiótica (temperatura elevada del compostaje).

Tabla 1

Caracterización Fisicoquímica del Biocarbón y Biocarbón Enriquecido

\begin{tabular}{lccccccccc}
\hline \multicolumn{1}{c}{ Muestra } & $\mathrm{H}(\%)$ & $\mathrm{pH}$ & $\mathrm{CE}(\mathrm{dS} / \mathrm{m})$ & $\mathrm{MO}(\%)$ & $\mathrm{C}(\%)$ & $\mathrm{N}(\%)$ & $\mathrm{P}(\%)$ & $\mathrm{K}(\%)$ \\
\hline Biocarbón & 15,28 & 7,85 & 0,72 & 5,43 & 55,32 & 0,63 & 0,23 & 0,54 \\
Biocarbón & & & & & & & & \\
enriquecido & 57,70 & 7,24 & 0,87 & 8,68 & 16,85 & 0,73 & 0,37 & 0,61 \\
\hline
\end{tabular}

Fuente: Laboratorio de Análisis de suelos, plantas, aguas y fertilizantes y Laboratorio de Fertilidad de SuelosUniversidad Nacional Agraria La Molina.

H: Humedad; pH: Potencial hidrógeno; CE: Conductividad eléctrica; MO: Materia orgánica; C: Carbono; N: Nitrógeno; P: Fósforo; K: Potasio.

Los valores obtenidos en el biocarbón enriquecido con respecto al biocarbón, difieren, así el contenido de $\mathrm{H}$ se ha incrementado debido a su inmersión en el biol, que tuvo por finalidad mejorar sus características fisicoquímicas, nutricionales y microbiológicas. El valor obtenido del pH disminuyó, puesto que los bioles de vacaza muestran siempre $\mathrm{pH}$ ácido (3,9 a 4,4;
Crespo, 2019), lo cual contribuyó a la ganancia de hidrógenos y una leve acidificación. La CE de se incrementó, como consecuencia de la liberación de sales a partir de la degradación de la materia orgánica en el biol y que el biocarbón, en función de su alta capacidad de intercambio catiónico (Wang y Wang, 2019), va a retener cationes, secuestrándolos en sus poros de 
manera constante, pero dicho valor concuerda con el obtenido por Pedroso-Reynaldo y PentónFernández (2019), quien activó el biochar en un lactofermentado con melaza, obteniendo una conductividad, menor de $1 \mathrm{dS} / \mathrm{m}$, que según Barbaro et al. (2014), es aceptable para un abono orgánico. Los valores de MO se incrementaron, debido a la adsorción de materia orgánica soluble procedente del biol (Méndez, 2017), que se traduce en el carbono fácilmente oxidable o de más rápida transformación (Méndez, 2017) presente en el biol, que comparado con 10,3 $\%$ obtenido en un biochar activado por cocompostaje en compost de vacuno, resulta ligeramente superior al biocarbón enriquecido por inmersión de biol de vacaza. El contenido de C disminuyó, debido a la actividad del consorcio microbiano presente en el biol, que hizo uso del carbono presente en el biocarbón, para suplir sus necesidades metabólicas, considerando que la inmersión del biocarbón se hizo por ocho días, tiempo suficiente para producirse la biodegradación del carbono presente en el biocarbón. El valor de $N$, sufrió un ligero incremento, como resultado de la migración del nitrógeno del biol a la estructura del biocarbón, y que resultó superior a 0,52 \% determinado por Mahmood et al. (2015), ligeramente superior al obtenido por Alonso-Gómez et al. (2016), de 0,7\% en promedio y un poco inferior al $1 \%$ obtenido por Liewet al. (2018), producto de las características de la materia prima como del suelo en el cual se cultivó la planta, condiciones edafoclimáticas en la cual se desarrolló el cultivo, condiciones de pirólisis como temperatura y tiempo de retención, por ello, Tortosa (2015) manifiesta que la disminución del nitrógeno se debe al efecto de la combustión de la biomasa (cuescos). El valor del P se incrementó, por la retención del P del biol en el biocarbón mediante adsorción física, también por interacciones químicas como intercambio iónico, atracción electrostática, precipitación y redox (Zhang et al., 2020), además el biocarbón proporciona superficies reactivas donde los iones P se retienen (Gul y Whalen, 2016), siendo su valor inferior a 0,91 obtenido por Parvage et al. (2013), que obtuvo biocarbón de residuos de trigo. El contenido de K, aumentó por efecto de la adsorción del K, que se infiere sea también por adsorción física o interacciones químicas, el cual resultó menor que 1,81\% reportado por Parvage et al. (2013), también para biocarbón de trigo, siendo de igual modo menor al valor de $2,4 \%$ en promedio indicado por Domínguez et al. (2020) para biocarbón de cuescos de palma.

\section{Tabla 2}

Resultados de la Caracterización Fisicoquímica del Suelo de las Parcelas Experimentales

\begin{tabular}{ccccccccc}
\hline Bloque & $\mathrm{pH}$ & $\begin{array}{c}\mathrm{MO} \\
(\%)\end{array}$ & $\begin{array}{c}\mathrm{DA} \\
\left(\mathrm{g} / \mathrm{cm}^{3}\right)\end{array}$ & $\begin{array}{c}\mathrm{CIC} \\
(\mathrm{meq} / 100 \mathrm{~g})\end{array}$ & $\begin{array}{c}\mathrm{N} \\
(\%)\end{array}$ & $\begin{array}{c}\mathrm{P} \\
(\mathrm{ppm})\end{array}$ & $\begin{array}{c}\mathrm{K} \\
(\mathrm{ppm})\end{array}$ & $\begin{array}{c}\text { Clase } \\
\text { textural }\end{array}$ \\
\hline $\mathrm{I}$ & 5,00 & 0,67 & 1,52 & 8,80 & 0,001 & 5,30 & 71,00 & Franco \\
II & 5,27 & 1,90 & 1,43 & 7,68 & 0,002 & 4,10 & 63,00 & Franco \\
III & 5,51 & 1,72 & 1,42 & 8,00 & 0,001 & 3,70 & 43,00 & Franco \\
IV & 5,57 & 2,86 & 1,36 & 12,32 & 0,002 & 3,70 & 56,00 & Franco \\
\hline
\end{tabular}

Fuente: Laboratorio de Análisis de suelos, plantas, aguas y Fertilizantes y Laboratorio de Fertilidad de SuelosUniversidad Nacional Agraria La Molina.

pH: Potencial hidrógeno; MO: Materia orgánica; DA: Densidad aparente; CIC: Capacidad de intercambio catiónico; N: Nitrógeno; P: Fósforo; K: Potasio. 
Los valores de $\mathrm{pH}$ obtenidos, corresponde a suelos muy ácidos (FAO, 2020a) con baja fertilidad, que, dependiendo de su grado de drenaje, pueden ser fluvisoles o gleisoles, típicos de Selva Baja conocida como la Región acrisólica ondulada (MIDAGRI, 2020). El porcentaje de MO presentado por los suelos de las parcelas, según Molina (2007), los ubicó en la categoría de Bajo a Medio que está por debajo del valor típico de suelos de Trópico que presentan un $5 \%$, conformado por material orgánico vivo o muerto, así en la parte viva se encuentran las raíces de las plantas, bacterias, lombrices de tierra, algas, hongos, en la parte muerta están los restos en descomposición de hojarascas, madera, estiércol (FAO, 2020b). La DA mostró valores propios de un suelo de textura franco, según Flores y Alcalá (2010), cuyo valor promedio de 1,43 g/ $\mathrm{cm}^{3}$ correspondiente al rango $1,35-1,5 \mathrm{~g} / \mathrm{cm}^{3}$, es un buen indicador de propiedades importantes del suelo, como: la compactación, porosidad, grado de aireación y capacidad de infiltración, que hacen posible la circulación de agua y aire en el suelo, los procesos de establecimiento de las plantas (germinación y enraizamiento) y del manejo del suelo (Rubio, 2010). Los datos de la CIC resultaron por debajo de 15 meq/100 g, según Yanez (1989) corresponde a suelos francos, y que reflejan su capacidad para retener bases, pues al ser un indicativo de su carga neta negativa, cuantifica el total de los cationes que pueden ser intercambiados entre la superficie de los coloides y la solución del suelo (Sumner y Miller, 1996) e indican la fertilidad de un suelo, así, valores por debajo de $5 \mathrm{meq} / 100 \mathrm{~g}$, resultan en suelos poco fértiles y por encima de $30 \mathrm{meq} / 100$ $g$, se trata de un suelo excesivamente arcilloso o con mucha MO, donde la planta corre peligro de sufrir asfixia radicular por encharcamiento (Yanez, 1989). Los valores del N, según Villarroel (1988), califican al suelo como Muy Bajo en este macronutriente, mientras Villasanti et al. (2013), establece que un suelo que muestre valores de $\mathrm{N}$ entre 0,00-0,10 ppm, se clasifica como Muy pobre, siendo inferiores a 0,25\% reportado por Medina (2019). La cantidad de P determinado, clasificó al suelo en Muy Bajo a Bajo según Villarroel (1988) y también fueron menores a 120000 ppm indicado por Villasanti et al. (2013), por lo cual ambos autores, califican al suelo Bajo en fósforo, que según Díaz (2020), es una característica de los suelos amazónicos, pues muestran una marcada pobreza en fósforo aprovechable. El análisis del contenido de K, que resultaron < 98 ppm establecido por Villarroel (1988), que clasificó la muestra de suelo como Muy Bajo en potasio, así también resultaron por debajo de 72 - 99 ppm de potasio disponible determinados por Casas (2014) en suelos de Yarinacocha-Pucallpa. La clase textural determinado clasificó al suelo muestreado como Franco, propio de terrazas medias, con contenidos de arena, limo y arcilla más o menos en la misma proporción (Casas, 2014).

\section{Aplicación de la enmienda y sus efectos en las características del suelo}

La enmienda se aplicó en dosis de 5, 10 y 15 t $h^{-1}(T 1, T 2$ y $T 3$ respectivamente), la cual es una práctica que ha sido realizada por Vásquez y Loly (2018), que utilizaron vermicompost en comparación con compost, de procedencia común, preparados en base de residuos de podas de jardín y estiércol vacuno para mejorar un suelo explotado por dieciocho años con monocultivo de Gypsophila (Gypsophila paniculata), usando dosis 0,25\%, 0,50\%, 1,00\%, 2,00 \% de enmienda en invernadero, logrando reducir la densidad, incrementar el contenido de $\mathrm{MO}$ y la reducción del pH. También Aróstegui (2019), evaluó la capacidad remediadora del biocarbón producido a partir de residuos agrícolas de sacha inchi y cacao sobre la mejora de las características físicas y químicas del suelo degradado por la agricultura intensiva de la región Ucayali, usando la especie Zea mays como indicadora, y reportó que la dosis del 5\% (la menor) fue la que mejores resultados arrojó, pues generó un pH neutro y mayor crecimiento de la planta.

\section{Análisis de varianza del potencial hidrógeno (pH)}

La Figura 1, muestra los resultados del análisis de varianza del $\mathrm{pH}$ de los suelos de las parcelas, que estadísticamente no muestran diferencia significativa $(p>0,05)$ entre los tratamientos, pero si a nivel de bloques, pues el bloque III, mostró un mayor valor el $\mathrm{pH}$, tal como reporta 
Aker (2014), quien aplicó biocarbón con gallinaza, logró incrementar el pH del suelo en 7,36 por disminución del Aluminio (Al) intercambiable lo cual tiende a reducir la acidez (Yamato et al., 2006).

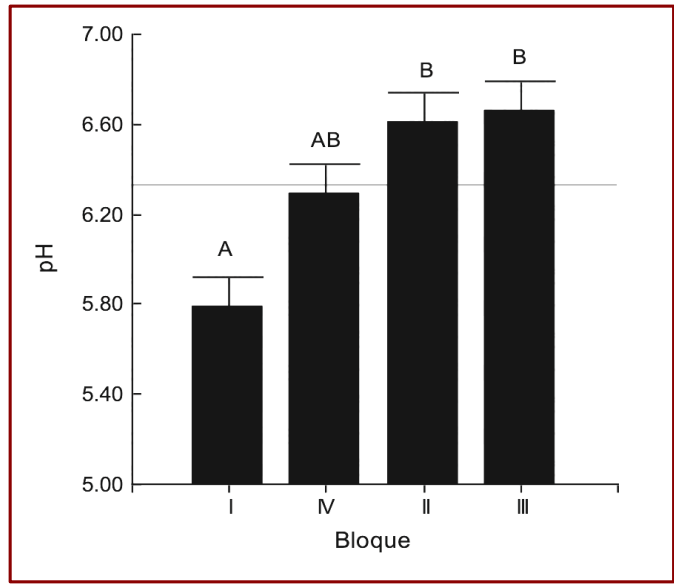

Figura 1. Efecto de los tratamientos en el pH del suelo por aplicación de biocarbón enriquecido.

Análisis de varianza de materia orgánica (MO) La MO, tanto a nivel de tratamientos y bloques (Tabla 3), no mostró diferencias significativas ( $p$ > 0,05); a pesar que autores como Méndez (2017), indican que incrementa el contenido de $\mathrm{MO}$, pero está en función del grado de oxidabilidad de la materia orgánica del biocarbón (Paco, 2012), el cual se ve influenciado por el tiempo que, al ser corto, no ha sufrido degradación y por consiguiente no se dio el incremento de $\mathrm{MO}$ en el suelo como producto de esta oxidación.

Tabla 3

Análisis de varianza de la materia orgánica (MO)

\begin{tabular}{lcrccc}
\hline \multicolumn{1}{c}{ F.V. } & SC & gl & CM & F & p-valor \\
\hline Modelo & 1,08 & 6 & 0,18 & 2,09 & 0,1537 \\
Tratamiento & 0,10 & 3 & 0,03 & 0,38 & 0,7666 \\
Bloque & 0,98 & 3 & 0,33 & 3,80 & 0,0520 \\
Error & 0,77 & 9 & 0,09 & & \\
Total & 1,86 & 15 & & & \\
\hline
\end{tabular}

FV: Fuente de variación; SC: Suma de cuadrados; gl: Grados de libertad; CM: Cuadrado medio; F: Prueba F.

\section{Análisis de varianza de densidad aparente (DA)}

La Tabla 4, corresponde a la densidad aparente, donde no se apreció los efectos de los tratamientos ni de los bloques, no existiendo diferencias significativas ( $p>0,05$ ); pero Aker (2014), disminuyó hasta $0,82 \mathrm{~g} / \mathrm{cm}^{3}$ aplicando biocarbón con gallinaza al suelo. Delayeet al. (2020), logró disminuir la DA de 1,31 a 1,26 g/ cm3, por el aumento en el suelo de su porosidad interna la cual se incrementó y la densidad aparente disminuyó (Oguntunde et al., 2008), infiriendo que está en función directa al tamaño de las partículas del biocarbón, es decir que partículas más grandes, generan mayor porosidad y partículas pequeñas, menor porosidad.

\section{Tabla 4}

Análisis de varianza de la densidad aparente (DA)

\begin{tabular}{lcrccc}
\hline \multicolumn{1}{c}{ F.V. } & SC & gl & CM & F & p-valor \\
\hline Modelo & 0,00089 & 6 & 0,00015 & 0,76 & 0,6199 \\
Tratamiento & 0,00042 & 3 & 0,00014 & 0,72 & 0,5672 \\
Bloque & 0,00047 & 3 & 0,00016 & 0,80 & 0,5241 \\
Error & 0,0018 & 9 & 0,00020 & & \\
Total & 0,0026 & 15 & & & \\
\hline
\end{tabular}

FV: Fuente de variación; SC: Suma de cuadrados; gl: Grados de libertad; CM: Cuadrado medio; F: Prueba F.

\section{Análisis de varianza de la capacidad de intercambio catiónico (CIC)}

La prueba no paramétrica de Friedman, en cuanto a la CIC de los suelos, indicó que no existió diferencia significativa $(p>0,05)$ del efecto de los tratamientos; pues la actividad del biocarbón en el suelo podría tener una importancia a largo plazo para sus procesos biogeoquímicos, que generarían aumentos en la capacidad de intercambio de cationes en relación con el ciclo de nutrientes, y que estaría en función de la polaridad que muestra; pues las cargas negativas presentes en el biocarbón facilitarían la adsorción de metales (Liang et al., 2006).

\section{Análisis de varianza del nitrógeno $(\mathrm{N})$}

El efecto de los tratamientos para mejorar el contenido de $\mathrm{N}$ de los suelos por la aplicación 
del biocarbón enriquecido (Figura 2), no mostró diferencias significativas ( $p>0,05$ ), pero a nivel de bloques, si se observó que el bloque IV mejoró en su contenido de N. Otras investigaciones demostraron un incremente de $\mathrm{N}$ del suelo, como el de Dumler (2019) que usó una enmienda de biocarbón con gallinaza, y Cedeño y Yeomans (2011), que experimentaron con compost+biocarbón, atribuyendo a su mineralización por efecto multiplicador de los microorganismos del compost y los atributos del biocarbón (retención de humedad, aporte de nutrientes, estabilidad del pH, hábitat favorable, etc.).

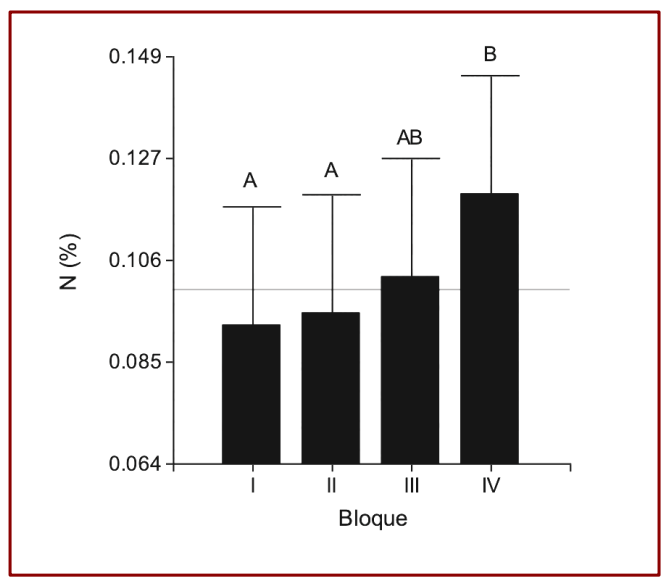

Figura 2. Efecto de los tratamientos en el $\mathrm{N}$ del suelo por aplicación de biocarbón enriquecido

\section{Análisis de varianza del fósforo (P)}

La Prueba de Friedman, aplicada para determinar el contenido de P (Tabla 5) en los suelos, mostró diferencia significativa $(p<0,05)$, destacando el T3 (15 t h h $^{-1}$ de biocarbón enriquecido) que aportó mayor P. Fiallos-Ortega et al. (2015), reportaron lo contrario, pues agregaron $30 \mathrm{t} \mathrm{h}^{-1}$ de carbón vegetal a un suelo para cultivo de alfalfa, su contenido de P descendió de 601,39 a 592,59 ppm, considerado significativo. Xu et al., (2013), reportaron que la aplicación de biocarbón mejoró la disponibilidad de $\mathrm{P}$, debido a los cambios de pH y la liberación directa del $\mathrm{P}$ del biocarbón al suelo, que al ser enriquecido con el P del biol, su aporte fue mayor, tal como se muestra en la presente investigación. Gilces (2014), reportó un incremento en el $\mathrm{P}$ asimilable del suelo cuando agregó biocarbón + cenizas.
Tabla 5

Análisis de varianza del fósforo (P)

\begin{tabular}{cccllll}
\hline Tratamiento & Suma (Ranks) & Media (Ranks) & $\mathrm{n}$ & & \\
\hline T0 & 5,00 & 1,25 & 4 & $\mathrm{a}$ & & \\
T1 & 7,00 & 1,75 & 4 & $\mathrm{a}$ & $\mathrm{b}$ & \\
T2 & 12,00 & 3,00 & 4 & & $\mathrm{C}$ & \\
T3 & 16,00 & 4,00 & 4 & & & $\mathrm{~d}$ \\
\hline
\end{tabular}

Medias con una letra común no son significativamente diferentes $(p>0.05)$

\section{Análisis de varianza del potasio (K)}

El análisis de varianza del K (Figura 3) a nivel de tratamientos indicó diferencias significativas $(p<$ $0,05)$, por lo cual la prueba de Tukey $(p<0,05)$ mostró al tratamiento $2\left(10 \mathrm{t} \mathrm{h}^{-1}\right.$ de biocarbón enriquecido) como el que mejoró el contenido de $\mathrm{K}$ del suelo, mientras Fiallos-Ortegaet al. (2015) reportó un descenso de 627,19 a 624,20 ppm, al agregar carbón vegetal a un suelo para cultivo de alfalfa. Guerra (2015), indica que el biocarbón producido a temperaturas $<700^{\circ} \mathrm{C}$, conserva $\mathrm{K}$, tal como se hizo en el presente estudio, el cual ha sido incrementado por el K del biol y luego retenido por la materia orgánica del suelo que presenta cargas negativas a través de un intercambio aniónico, pues el K posee carga positiva, incrementando así el contenido de K del suelo intervenido.

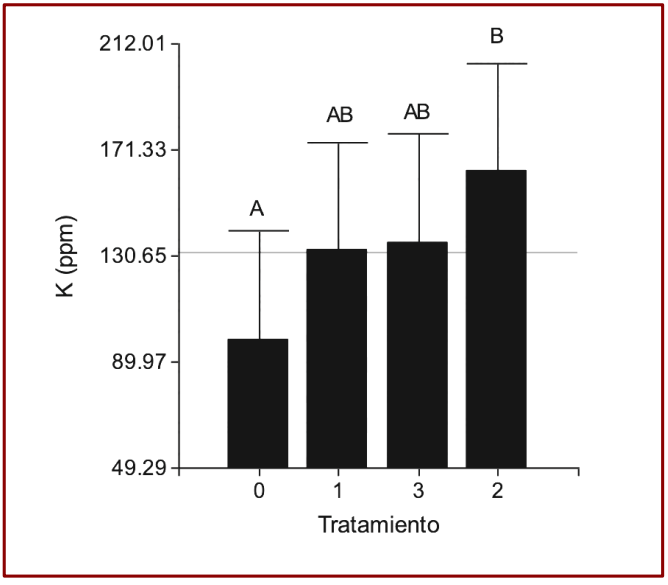

Figura 3. Efecto de los tratamientos en el K del suelo por aplicación de biocarbón enriquecido. 


\section{CONCLUSIONES}

La aplicación de biocarbón enriquecido en biol de vacaza, causaron efectos estadísticamente significativos de mejoramiento en las características del suelo sobrepastoreado en el contenido de $\mathrm{P}$ y $\mathrm{K}$, lo cual no ocurrió con el $\mathrm{pH}$, $\mathrm{MO}, \mathrm{DA}, \mathrm{CIC}, \mathrm{N}$, donde no se observaron efectos significativos de mejora, lo cual en base a los resultados de investigaciones desarrolladas para demostrar su actividad mejoradora del suelo, este es un proceso lento y que dependen de factores como tipo y uso del suelo, tipo de sembríos y factores edafoclimáticos, lo cual asegura que en los próximos años, se hará notorio sus beneficios en la recuperación de las características agrícolas de los suelos intervenidos, pues el biol le confiere propiedades fisicoquímicas basados en su capacidad adsorbente de sus poros externos, para formar una capa nutricional en su superficie y absorbente de sus poros internos, para retener los nutrientes del biol e ir distribuyéndolos a lo largo del tiempo, beneficiando al consorcio de microorganismos, suelo y plantas, por ello el comportamiento del biocarbón enriquecido en el suelo aún necesita mayores estudios.

\section{AGRADECIMIENTOS}

- A la Agencia Española de Cooperación Internacional para el Desarrollo (AECID) por su participación activa para la ejecución de las investigaciones desarrolladas en las regiones de Apurímac y Ucayali.

- Al Instituto de la Pequeña Producción Sustentable (IPPS) por el financiamiento, asesoramiento, difusión y apoyo constante en el desarrollo de la investigación.

- Al Dr. Mariano Magdaleno Mendoza Carlos, por permitirnos utilizar las instalaciones de su fundo para el desarrollo de la presente investigación.

\section{REFERENCIAS BIBLIOGRÁFICAS}

Aker Narváez, C. E. (2014). Evaluación del uso de estufas ecológicas, análisis de la producción y uso del biocarbón como enmienda del suelo en los rendimientos de maíz (Zea mays) en tres municipios de León, Nicaragua [Tesis de maestría, Centro Agronómico Tropical de Investigación y Enseñanza]. http://201.207.189.89/ bitstream/handle/11554/7130/Evaluacion_ del_uso_de_estufas_ecologicas. pdf? sequence $=1 \&$ isAllowed $=y \quad$ Disponible en: $\quad$ http://201.207.189.89/bitstream/ handle/11554/7130/Evaluacion_ del_uso_de_estufas_ecologicas. pdf? sequence $=1$ \&isAllowed $=\mathrm{y}$.

Alonso-Gómez, L., Cruz-Dominguez, A., JiménezMadrid, D., Ocampo-Duran, Á., \& ParraGonzález, S. (2016). Biochar como enmienda en un oxisol y su efecto en el crecimiento de maíz. Ciencias de la Salud, 341. doi: https:// doi.org/10.31910/rudca.v19.n2.2016.88

Arévalo Maldonado, E. N. (2020). Valoración de la calidad del suelo mediante la aplicación de biochar de acícula de pino (Pinus patula) en la cuenca del MachángaraSaucay [Tesis de titulación, Universidad Politécnica Salesiana].https://dspace. ups.edu.ec/handle/123456789/19180. Disponible en: https://dspace.ups.edu.ec/ handle/123456789/19180.

Aróstegui Cornelio, K. (2019). Evaluación del efecto de biochar de residuos de sacha inchi y cacao sobre suelos degradados de Campoverde, Ucayali, usando Zea mays como indicador [Tesis de titulación, Universidad Científica del Sur]. $\quad$ https://repositorio.cientifica.edu. pe/bitstream/handle/20.500.12805/762/ T L - A r \% C $3 \%$ B 3 s t e g u i \% $20 \mathrm{~K}$. pdf?sequence $=1 . \quad$ Disponible en: https://repositorio.cientifica.edu.pe/ bitstream/handle/20.500.12805/762/TLAr\%C3\%B3stegui\%20K.pdf?sequence=1.

Barbaro, L., Karlanian, M., \& Mata, D. (2014). Importancia del pH y la Conductividad Eléctrica (CE) en los sustratos para plantas. Presidencia de la Nación, Ministerio de Agricultura, Ganadería y Pesca, Instituto Nacional de Tecnología Agropecuaria (INTA). Argentina. Disponible en: https://inta.gob. ar/sites/default/files/script-tmp-inta_-_ 
importancia_del_ph_y_la_conductividad_ elctrica.pdf

Casas Vera, J. H. (2014). Curva de absorción de nutrientes en la biomasa estacional del cultivo de camu camu (Myrciaria dubia HBK McVaugh) en suelos de Yarinacocha (Pucallpa) [Tesis de maestría, Universidad Nacional Agraria La Molina].http:// repositorio.lamolina.edu.pe/handle/ UNALM/2344. Disponible en: http:// repositor io.lamolina.edu.pe/handle/ UNALM/2344. Cedeño Cedeño, A. M

Cedeño Cedeño, A. M., \& Yeomans, J. (2011). Mineralización de nitrógeno en suelo con enmiendas de biofertilizantes (No. PG 20 2011).

Cochrane, T. T. (1982). Caracterización agroecológica para el desarrollo de pasturas en suelos ácidos de América tropical. Centro Internacional de Agricultura Tropical (CIAT).

Crespo Troya, A. A. (2019). Obtención de biogás utilizando como base el estiércol de ganado vacuno enla parroquia La Unión, cantón Babahoyo, provincia de Los Ríos [Tesis de título profesional, Universidad Técnica de Babahoyo]. Disponible en: http:// dspace.utb. edu.ec/bitstream/handle/ 49000/7264/ TE-UTB-FACIAG-ING\%20 AGROP-000097. pdf?sequence $=1$

Delaye, L. A. M., Ullé, J. Á., \& Andriulo, A. (2020). Aplicación de biochar en un suelo degradado bajo producción de batata. Efecto sobre propiedades edáficas. Ciencia del suelo, 38(1), 162-173. Disponible en: http://www.suelos.org.ar/publicaciones/ Volumen38n1/15-561.pdf

Díaz Gonzales, W. (2020). Cambios en las propiedades físicas, químicas,biológicas y captura de carbono del suelo en la recuperación de pasturas degradadas de braquiaria (Brachiaria decumbens), en Pucallpa, Perú [Tesis de título profesional, Universidad Nacional de Ucayali]. http:// repositorio.unu.edu.pe/bitstream/handle/ UNU/4617/UNU_AGRONOMIA_2020_T_ W I L L | A N - D | A Z - G O N Z A LES. pdf? sequence $=1$. Disponible en: http:// repositorio.unu.edu.pe/bitstream/handle/ UNU/4617/UNU_AGRONOMIA_2020_T_ WILLIAN-DIAZ-GONZALES.pdf? sequence=1.

Domínguez, E. L., Uttran, A., Loh, S. K., Manero, M. H., Upperton, R., Tanimu, M. I., \& Bachmann, R. T. (2020). Characterization of industrially produced oil palm kernel shell biochar and its potential as slow release nitrogenphosphate fertilizer and carbon sink. Materials Today: Proceedings. doi: https:// doi.org/10.1016/j.matpr.2020.05.143

Dumler Granthon, J. S. (2019). Efecto de biochar y gallinaza en el flujo de nitrógeno de un sistema suelo entisol - cultivo de maíz en San Ramón, Perú [Tesis de titulación, Universidad Científica del Sur]. https:// repositorio.cientifica.edu.pe/bitstream/ handle/20.500.12805/935/TL-Dumler\%20J. pdf? sequence $=1$. Disponible en: https:// repositorio.cientifica.edu.pe/bitstream/ handle/20.500.12805/935/TL-Dumler\%20J. pdf? sequence $=1$.

FAO (Organización de las Naciones Unidas para la Alimentación y la Agricultura). (21 de diciembre de 2020a). Portal de suelos de la FAO. Propiedades químicas. El pH del suelo. http://www.fao.org/soils-portal/soilsurvey/clasificacion-de-suelos/sistemasnumericos/propiedades-quimicas/ es/. Disponible en: http://www.fao.org/ soils-portal/soil-survey/clasificacion-desuelos/sistemas-numericos/propiedadesquimicas/es/.

FAO (Organización de las Naciones Unidas para la Alimentación y la Agricultura). (21 de diciembre de 2020b). Tema 4. Los suelos necesitan nutrirse:plantas, microorganismos, agua, aire y abonos. Disponible en : http://www.fao.org/3/ah645s/AH645S05. htm\#: :text=Los\%20suelos\%20del\%20 Tr\%C3\%B3 pico\%20de,les\%20dan\%20 
vida\%20y\%20fertilidad.\&text=Los\%20 organismos\%20del\%20suelo\%20y\%20 las\%20plantas\%20necesitan\%20agua\%20 para\%20vivir.

Fiallos-Ortega, L. R., Flores-Mancheno, L. G., Duchi-Duchi, N., Flores-Mancheno, C. I., Baño-Ayala, D., \& Estrada-Orozco, L. (2015). Restauración ecológica del suelo aplicando biochar (carbón vegetal), y su efecto en la producción de Medicago sativa. Ciencia y Agricultura, 12(2), 13-20. https://doi. org/10.19053/01228420.4349

Flores Delgadillo, L., Alcalá Martínes, J. R., (2010). Manual de Procedimientos Analíticos. Laboratorio de Física de Suelos. Disponible en: https:// www.geologia.unam.mx/igl/ deptos/edafo/ Ifs/MANUAL\%20DEL\%20 LABORATORIO\%20 DE\%2OFISICA\%20 DE\%20SUELOS1.pdf.

Flores Romayna, M. A., Ortega Chávez, W., \& Ortega Mallqui, A. (2020). Evaluación de tratamientos pregerminativos en semillas de Euterpe precatoria Mart.(Huasaí) en la ciudad de Pucallpa-Perú. Revista Cubana de Ciencias Forestales, 8(1), 88-103. Disponible en: http://scielo.sld.cu/pdf/cfp/v8n1/23103469-cfp-8-01-88.pdf

Gilces Reyna, M. A. (2014). Efectos de la aplicación de biochar y cenizas en las propiedades del suelo [Tesis de maestría, Universidad de Valladolid]. Disponible en: https://uvadoc.uva.es/ bitstream/handle/10324/6632/TFM-L190. pdf? sequence $=1$ \&isAllowed $=\mathrm{y}$.

Graber, E. R., Harel, Y. M., Kolton, M., Cytryn, E., Silber, A., David, D. R., \& Elad, Y. (2010). Biochar impact on development and productivity of pepper and tomato grown in fertigated soilless media. Plant and soil, 337(1-2), 481496.doi: https://doi.org/10.1007/s11104010-0544-6

Guachalá, V., \& Zeneida, G. (2020). Mejoramiento de la capacidad productiva en suelos degradados de unidades de producción agrícola en la comunidad Manzano Guaranguí, provincia de Imbabura [Tesis de maestría, Universidad Técnica de Cotopaxi (UTC)]. Disponible en: http://repositorio.utc. edu.ec/ bitstream/27000/5992/1/MUTC000654.pdf.

Guerra Laura, P. A. (2015). Producción y caracterización de Biochar a partir de la biomasa residual de sistemas agroforestales y de agricultura convencional en la Amazonía Peruana [Tesis de titulación, Universidad Nacional Agraria La molina]. disponible en: http://repositorio.lamolina. edu.pe/ bitstream/handle/UNALM/1895/ Q70.G84-T. pdf? sequence = 1\&isAllowed=y.

Gul, S., \&Whalen, J. K. (2016). Biochemical cycling of nitrogen and phosphorus in biocharamended soils. Soil Biology and Biochemistry, 103, 1-15. doi: 10.1016/ j.soilbio.2016.08.001

Hernández-Medrano, J. H., \& Corona, L. (2018). EEl metano y la ganadería bovina en México: ¿Parte de la solución y no del problema? Agroproductividad, 11(2), 46-51. Disponible en: $\quad$ https://core.ac.uk/download/ pdf/249319993.pdf

Huaraya Huahualuque, M. Y., \&Sancho Moya, T. C. (2020). Estado del arte sobre la producción de biogás mediante la digestión anaerobia como parte del aprovechamiento de la biomasa residual pecuaria [Tesis de bachillerato, Universidad Peruana Unión]. Disponible en: https://repositorio.upeu.edu. pe/bitstream/ handle/UPEU/3415/Mer y_ Trabajo_Bachiller_2020.pdf?sequence $=5$.

Hugill, B. (2013). Carbón Biológico-Un hogar Orgánico para Microbios del Suelo. ECHO Nota Técnica. 1(75),1-5. Disponible en: https://www.echocommunity.org/es/ resources/070b6c33-4118-43c2-a26caf58c60fe51a

Ippolito, J. A., Spokas, K. A., Novak, J. M., 
Lentz, R. D., \& Cantrell, K. B. (2015). Biochar elemental composition and factors influencing nutrient retention. Biochar for environmental management: Science, technology and implementation, 139-163. Disponible en: https://eprints.nwisrl.ars. usda.gov/id/eprint/1590/

Kim, WK, Shim, T., Kim, YS, Hyun, S., Ryu, C., Park, YK y Jung, J. (2013). Caracterización de la eliminación de cadmio de una solución acuosa mediante biocarbón producido a partir de un Miscanthus giganteus a diferentes temperaturas pirolíticas. Tecnología de fuentes biológicas, (138), 266270.

Klug, M. (2012). Pirólisis, un proceso para derretir la biomasa. Revista de Química, 26(12), 37-40. Disponible en: http://revistas. pucp.edu.pe/index.php/quimica/article/ view/5547/5543

Langone, M., Soldano, M., Fabbri, C., Pirozzi, F. y Andreottola, G. (2017). Digestión anaeróbica de estiércol de ganado influenciada por cavitación hidrodinámica inducida por chorros de remolino. Bioquímica y biotecnología aplicada, 184 (4), 1200-1218. doi: 10.1007 / s12010-017-2612-3.

Liang, B., Lehmann, J., Solomon, D., Kinyangi, J., Grossman, J., O'neill, B., ... \& Neves, E. G. (2006). Black carbon increases cation exchange capacity in soils. Soil Science Society of America Journal, 70(5), 1719-1730. Disponible en: http://xrm. phys.northwestern.edu/research/pdf_ papers/2006/liang_sssaj_2006.pdf

Liew, R. K., Nam, W. L., Chong, M. Y., Phang, X. Y., Su, M. H., Yek, P. N. Y., ... y Lam, S. S. (2018). Residuos de palma aceitera: una materia prima abundante y prometedora para la conversión por pirólisis de microondas en biocarbón de buena calidad con múltiples aplicaciones potenciales. Seguridad de procesos y protección ambiental, (115), 5769.
Mahmood, W. M. F. W., Ariffin, M. A., Harun, Z., Ishak, N. A. I. M., Ghani, J. A., Rahman, M. A. (2015). Caracterización y uso potencial de biocarbón de residuos de palma aceitera gasificada. Revista de ciencia y tecnología de la ingeniería, 10 (2014), 45-54.

Marín Saldada, M. E. (2019). Rendimiento y composición química de cuatro variedades de alfalfa (Medicago sativa) en Cajamarca [Tesis de título profesional, Universidad de Cajamarca] .Disponible en: http://190.116.36.86/handle/ UNC/3202.

Medina, C. A. B., Pérez, R. D. A., Almeida, J. A. F., Morán, H. F. R., Inmunda, M. W. A., Rojas, J. L. A., ... \& Ibarra, E. M. (2019). Evaluación del uso de un biocarbono sobre la absorción de cadmio del suelo y la productividad del cultivo de cacao (Theobroma cacao L.) en la Amazonía ecuatoriana. Revista Iberoamericana Ambiente \& Sustentabilidad, 6-15. doi: https://doi.org/10.46380/rias.v2i1.33

Méndez Fernández, A. (2017). Cambio en las propiedades químicas de unbiochar de sarmientos de vid por adición apilas de compostaje [Tesis de maestría, Universidad deValladolid]. Disponible en: https://uvadoc. uva.es/ bitstream/handle/10324/25146/ TFM-I-659. pdf? sequence $=1$.

MIDAGRI (Ministerio de Desarrollo Agrario y Riego). (21 de diciembre de 2020). Clasificación de los suelos. Disponible en: http://minagri. gob.pe/portal/datero/43sector-agrario/suelo/330-clasificacion.

Molina, E. (2007). Análisis de suelos y su interpretación. San José, CR, CIA-UCRAmino Grow International. Disponible en: $\quad$ http://www.infoagro.go.cr/ Inforegiones/RegionCentralOriental/ Documents/Suelos/SUELOSAMINOGROWanalisiseinterpretacion.pdf

Molina, E., Meléndez, G. (2002). Tabla de interpretación de análisis de suelos. Centrode Investigaciones Agronómicas, 
Universidad de Costa Rica. Mimeo.

Obregón Castro, G. D. P. (2019). Disminución de la salinidad de suelos aplicando biochar a base de biomasa animal y vegetal en Cañete, 2019 [Tesis de bachillerato, Universidad César Vallejo]. Disponible en: $\quad$ http://repositorio.ucv.edu.pe/ bitstream/handle/20.500.12692/35709/B_ O bre g \% C $3 \%$ B 3 n_ C G D P. pdf? sequence $=1$ \&isAllowed $=y$.

Oguntunde, P. G., Abiodun, B. J., Ajayi, A. E., \& van de Giesen, N. (2008). Effects of charcoal production on soil physical properties in Ghana. Journal of Plant Nutrition and Soil Science, 171(4), 591-596. doi: https://doi. org/10.1002/jpln.200625185

Paco Abenza, D. (2012). Evaluación de efectos de varios tiposde biochar en suelo y planta [Tesis de licenciatura, Universidad Autónoma de Barcelona]. Disponible en: https://ddd.uab.cat/pub/ trerecpro/2012/ hdl_2072_202695/PFC_DanielPacoAbenza. pdf.

Parvage, M. M., Ulén, B., Eriksson, J., Strock, J., \& Kirchmann, H. (2013). Phosphorus availability in soils amended with wheat residue char. Biology and fertility of soils, 49(2), 245-250. doi: https://doi.org/10.1007/s00374-0120746-6

Pedroso-Reynaldo, A., \& Pentón-Fernández, G. (2019). Efecto físico-químico que ejerce la fuente de energía de soluciones nutritivas sobre el biochar enriquecido [ponencia]. V Convención Internacional de Agrodesarrollo, Matanzas, Cuba. Disponible en: https://www.researchgate.net/ profile/ Biochar-Cuba/publication/337196551_ Efecto_fisico-quimico_que_ejerce_la_ fuente_de_energia_de_soluciones_ nutritivas_sobre_el_biochar_enriquecido/ links/5dcadab292851c818049aea1/Efectofisico-quimico-que-ejerce-la-fuente-deenergia-de-soluciones-nutritivas-sobre-elbiochar-enriquecido.pdf.
Pérez Espejo, A. M. (2009). Efecto de la fertilización foliar orgánica a base de bioles en la producción de camu camu (Myrciaria dubia H.B.K Mc Vaugh) en un entisol de Pucallpa [Tesis de título profesional, Universidad Nacional de Ucayali]. Disponible en: http://181.176.160.68/ bitstream/handle/UNU/2111/000001580T. pdf? sequence $=1$ \&isAllowed $=\mathrm{y}$.

Plant Health Cure BV. (6 de diciembre de 2017). PHC Película: El suelo es un organismo viviente [Archivo de Vídeo]. Disponible en: https://www.youtube.com/ watch?v=gJOiEbdFURE\&feature $=e m b$ logo.

Prost, K., Borchard, N., Siemens, J., Kautz, T., Séquaris, JM, Möller, A. y Amelung, W. (2013). Biocarbón afectado por compostaje con estiércol de corral. Revista de calidad ambiental, 42 (1), 164-172.

Remigio Ipanaque, S. R. (2018). Uso del biochar de excretas porcina y avícola en la reducción de cadmio ensuelo agrícola contaminado, Huaral, 2018 [Tesis de titulación, Universidad César Vallejo]. Disponible en: http://repositorio.ucv.edu. pe/bitstream/ handle/20.500.12692/21230/ Remigio_ISR. pdf? sequence $=4$

Rojas-Pérez, F., Palma-López, D., Salgado-García, S., Obrador-Olán, J. J., Arreola-Enríquez, J. (2020). Elaboración y caracterización nutrimental de abonos orgánicos líquidos en condiciones tropicales. Agroproductividad, 13(4), 73-78. Disponible en: https://www.researchgate.net/ profile/ David_Palma3/publication/342146589_E I a boracion_y_caracterizaci o n _ nutrimental_de_abonos_organicos_ liquidos_en_condiciones_tropicales/ links/5ef24b47299bf1031f1c0230/ Elaboracion-y-caracterizacion-nutrimentalde-abonos-organicos-liquidos-encondiciones-tropicales.pdf.

Rubio Gutiérrez, A. M. (2010). La densidad 
aparente en suelos forestales del parque natural los alcornocales [Trabajo de titulación, Universidad de Sevilla]. Disponible en: https:// digital.csic. es/bitstream/10261/57951/1/La\%20 densidad $\% 20$ aparente $\% 20$ en $\% 20$ suelos\%20forestales\%20.pdf.

Sandoval, V. P., Jaca, C., \& Ormazabal, M. (2017). Economía circular. Memoria Investigaciones en Ingeniería, (15), 85-95. Disponible en: http://revistas.um.edu.uy/index.php/ ingenieria/article/view/308/366

Sumner, M. E., \& Miller, W. P. (1996). Cation exchange capacity and exchange coefficients. In D.L. Sparks A.L. Page P.A. Helmke R.H. Loeppert P. N. Soltanpour M. A. Tabatabai C. T. Johnston M. E. Sumner (Ed.), Methods of Soil Analysis: Part 3 Chemical Methods, 5.3, (pp. 1201-1229). Wisconsin, EE.UU:: Soil Science Society of America, Inc., American Society of Agronomy, Inc. Serie de libros SSSA. doi: https://doi.org/10.2136/ sssabookser5.3.c40

Suquilanda, M. (1996). Agricultura orgánica, alternativa tecnológica del futuro. Fundagro, Quito.

Tzvetkov, G., Mihaylova, S., Stoitchkova, K., Tzvetkov, P. y Spassov, T. (2016). Activación mecanoquímica y química de material lignocelulósico para preparar carbones activados en polvo para aplicaciones de adsorción. Tecnología en polvo, (299), 41-50.

Uribe Calderón, J. (2020). Nanomateriales [Diapositivas de Power Point]. Recuperado de: Curso Virtual en el Marco de la Ejecución del Proyecto: "Obtención y caracterización de nanocristales de almidón a partir de papas nativas y de tunta, empleando métodos amigables con el ambiente". Contrato No. 153-2017-FONDECYT. Dictado del 23 al 27 de noviembre del 2020. www. lamolina.edu. pe/industrias.

Vázquez, J., \& Loli, O. (2018). Compost y vermicompost como enmiendas en la recuperación de un suelo degradado por el manejo de Gypsophila paniculata. Scientia Agropecuaria, 9(1), 43-52. doi: http://dx.doi. org/10.17268/sci.agropecu.2018.01.05.

Villasanti, C., Román, P., \& Pantoja, A. (2013). El manejo del suelo en la producción de hortalizas con buenas prácticas agrícolas. Paraguay: FAO. Disponible en: .http://www. fao.org/3/a-i3361s.pdf.

Villarroel, J. (1988). Manual práctico para la interpretación de análisis de suelos en laboratorio. Disponible en: http:// atlas.umss.edu.bo:8080/xmlui/ bitstream/handle/123456789/142/ MANUAL\%2 OPRACTICO\%20ST 10. pdf? sequence $=1$ \&isAllowed $=y$

Wang, J., \& Wang, S. (2019). Preparation, modification and environmental application of biochar: a review. Journal of Cleaner Production, (227), 1002-1022. doi: https:// doi.org/10.1016/j.jclepro.2019.04.282

Xu, G., Wei, L. L., Sun, J. N., Shao, H. B., \& Chang, S. X. (2013). What is more important for enhancing nutrient bioavailability with biochar application into a sandy soil: Direct or indirect mechanism?. Ecological Engineering, (52), 119-124. doi: https://doi. org/10.1016/j.ecoleng.2012.12.091

Yamato, M., Okimori, Y., Wibowo, IF, Anshori, S. y Ogawa, M. (2006). Efectos de la aplicación de la corteza carbonizada de Acacia mangium sobre el rendimiento de maíz, caupí y maní, y las propiedades químicas del suelo en el sur de Sumatra, Indonesia. Ciencia del suelo y nutrición vegetal, 52(4), 489-495. doi: https://doi. org/10.1111/j.1747-0765.2006.00065.x

Yanez Jiménez, J. (1989). Análisis de suelos y su interpretación. Horticultura: Revista de industria, distribución y socio-economía hortícola: frutas, hortalizas, flores, plantas, árboles ornamentales y viveros, (49), 75-89. 
Disponible en: https://www.mapa.gob.es/ ministerio/ pags/biblioteca/revistas/pdf_ Hort/Hort_1989_49_75_89.pdf

Zhang, A., Li, X., Xing, J., \& Xu, G. (2020). Adsorption of potentially toxic elements in water by modified biochar: A review. Journal of Environmental Chemical Engineering, 104196. doi: https://doi.org/10.1016/j. jece.2020.104196 